\title{
Mathematical and Numerical Analysis of Heat Transfer Enhancement by Distribution of Suction Flows inside Permeable Tubes
}

\author{
A.-R. A. Khaled \\ Mechanical Engineering Department, King Abdulaziz University, P.O. Box 80204, Jeddah 21589, Saudi Arabia
}

Correspondence should be addressed to A.-R. A. Khaled; akhaled4@yahoo.com

Received 1 February 2015; Revised 24 March 2015; Accepted 27 March 2015

Academic Editor: Rama S. R. Gorla

Copyright (C) 2015 A.-R. A. Khaled. This is an open access article distributed under the Creative Commons Attribution License, which permits unrestricted use, distribution, and reproduction in any medium, provided the original work is properly cited.

\begin{abstract}
Heat transfer enhancement in permeable tubes subjected to transverse suction flow is investigated in this work. Both momentum and energy equations are solved analytically and numerically. Both solutions based on negligible entry regions are well matched. Two different suction velocity distributions are considered. A parametric study including the influence of the average suction velocity and the suction velocity profile is conducted for various Peclet numbers. It is found that enhancement of heat transfer over that in impermeable tubes is only possible with large Peclet numbers. This enhancement increases as suction velocities towards the tube outlet increase and as those towards the tube inlet decrease simultaneously. The identified enhancement mechanisms are expanding the entry regions, increasing the transverse advection, and increasing the downstream excess temperatures under same transverse advection. The average suction velocity that produces maximum enhancement increases as the Peclet number increases until it reaches asymptotically its uppermost value at large Peclet numbers. The maximum reported enhancement ratios for the exponential and linear suction velocity distributions are 17.62-fold and 14.67-fold above those for impermeable tubes, respectively. This work demonstrates that significant heat transfer enhancement is attainable when the suction flow inside the permeable tubes is distributed properly.
\end{abstract}

\section{Introduction}

The study of fluid flow and heat transfer inside permeable tubes or channels exposed to surface suction is important to many industrial applications. These applications include transpiration cooling where channel surfaces are cooled by passing cooled fluid through the pores of these surfaces, controlling boundary layers over surfaces of airplane wings or turbine blades by injection or suction of fluid at theses surfaces, lubrication of permeable bearings, fluid filtration processes, cooling of combustion chambers exhaust ports, and cooling of porous walled reactors [1]. Accordingly, the aim of the present work is to investigate heat transfer enhancement inside permeable tubes subjected to nonuniform surface suction.

Bergles [2] indicated that surface suction is an effective technique that can be used to enhance heat transfer $[2,3]$. He indicated that improvement in heat transfer coefficient is expected to reach several hundred percent for laminar flow with suction at the solid boundary [2-5]. He pointed out that this enhancement is due to reduction in the boundary layer thickness [2]. This can be clearly seen for external flows [4-8]. However, surface suction within internal flows tends to reduce the mean velocity inside the tube or the channel. This effect may thicken the boundary layer and causes impediments of both flows near the boundary and heat transfer rate. Therefore, the novelty of the present work is to explore the various conditions that may reveal heat transfer enhancement inside permeable tubes exposed to surface suction.

Among initial works that analyzed the problem of fluid flow and heat transfer inside permeable tubes or channels with surface suction are the works of Kinney [9], Pederson and Kinney [10], Raithby [11], and Sorour and Hassab [12]. These works illustrated the variations of the temperature profile and Nusselt number with wall Reynolds number in 
porous tubes or channels. These works and many others were the motivations behind recent works that accounted for all possible hydrodynamic conditions on heat transfer inside channels subjected to wall suction. For example, Sorour et al. [13] and Bubnovich et al. [14] analyzed dynamically and thermally the developing flow inside a channel subjected to nonuniform suction at one wall. Hwang et al. [15] investigated numerically forced laminar convection in the entrance region of a square duct subjected to uniform mass transpiration. Makinde et al. [1] analyzed heat transfer in channels exposed to wall suction in presence of nanofluids with both wall slip and viscous dissipation effects. All of the aforementioned works and many similar ones in the literature did not explore the heat transfer enhancement due to surface suction inside permeable tubes. Thus, the motivation behind the present work is to enrich the literature with a study about the role of surface suction and its profile inside permeable tubes on heat transfer enhancement.

Promoting flow close to the energy exchanging boundary usually results in heat transfer enhancement $[2,16]$. Meanwhile, the internal flow impedance near this boundary increases as suction Reynolds number $\left(\operatorname{Re}_{v_{w}}\right)$ increases [9, 11]. For permeable circular tubes, the internal flow starts to separate from the boundary when $\mathrm{Re}_{v_{w}}=4.5978$ due to the adverse pressure gradient caused by reduction in the mean fluid velocity. To avoid this instability condition and to sustain maximum velocity close to tube surface, the suction Reynolds number must be $\operatorname{Re}_{v_{w}} \leq 1.04$. This constraint can be shown using Kinney [9] and Raithby [11] works to yield universal surface friction coefficient to be at least equal to $95 \%$ of its maximum value when $\operatorname{Re}_{v_{w}}=0$. Accordingly, the flow close to the energy exchanging boundary can be kept maximally promoted under this constraint. Therefore, the present work is concerned with heat transfer enhancement inside permeable tubes exposed to surface suction with $0<\operatorname{Re}_{v_{w}} \leq 1.04$.

In the next sections, flow and heat transfer inside a preamble tube subjected to internal suction flow are modelled and analyzed. The surface suction velocity is considered to have either linear or exponential profile distributions. Both momentum and thermal energy transfer equations are solved using various analytical and numerical methods. Different heat transfer enhancement indicators are computed. Both analytical and numerical computations of these indicators are validated under an applicable constraint and using early studies. An extensive parametric study has been conducted in order to identify and explore the influence of average suction velocity, suction velocity profile, and Peclet number on the heat transfer enhancement indicators.

\section{Problem Formulation}

2.1. Modeling of Flow and Heat Transfer inside the Permeable Tube. Consider a tube of length $L$ and inner diameter $D$. The tube wall is permeable so as to allow fluid suction at its boundary as shown in Figure 1. Both the flow inside the tube and that through the permeable boundary are considered to be laminar flows. The density, specific heat, thermal conductivity, and the dynamic viscosity of the fluid are $\rho, c_{p}, k$, and $\mu$, respectively. The dimensionless continuity,



(a)

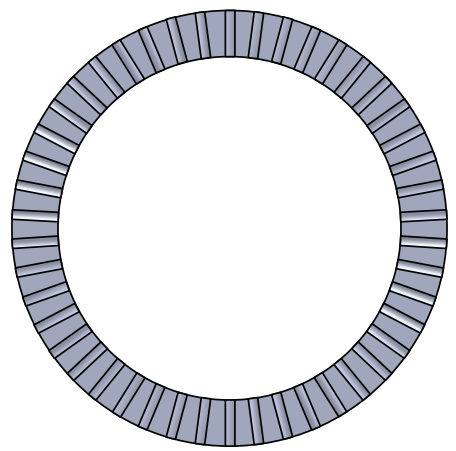

(b)



(c)

FIGURE 1: (a) 3D view of the tube with suction passages embedded on its material volume, (b) cross section of the tube, and (c) schematic profile of the tube and the coordinates system.

momentum, and energy equations of the fluid are given by [17-19]:

$$
\begin{gathered}
\frac{\partial \bar{u}}{\partial \bar{x}}+\frac{1}{\bar{r}} \frac{\partial}{\partial \bar{r}}(\bar{r} \bar{v})=0 \\
a \operatorname{Re}_{r}\left(\bar{u} \frac{\partial \bar{u}}{\partial \bar{x}}+\bar{v} \frac{\partial \bar{u}}{\partial \bar{r}}\right)=16 \frac{d \bar{p}}{d \bar{x}}+\frac{2}{\bar{r}} \frac{\partial}{\partial \bar{r}}\left(\bar{r} \frac{\partial \bar{u}}{\partial \bar{r}}\right), \\
a \operatorname{Pe}_{r}\left(\bar{u} \frac{\partial \theta}{\partial \bar{x}}+\bar{v} \frac{\partial \theta}{\partial \bar{r}}\right)=\frac{2}{\bar{r}} \frac{\partial}{\partial \bar{r}}\left(\bar{r} \frac{\partial \theta}{\partial \bar{r}}\right),
\end{gathered}
$$


where $\bar{u}$ and $\bar{v}$ are the dimensionless axial and radial velocities, respectively. $\bar{x}$ and $\bar{r}$ are the dimensionless axial and radial positions, respectively. $a$ and $\mathrm{Pe}_{r}$ are the tube aspect ratio and the reference Peclet number, respectively. $\bar{p}$ and $\theta$ are the dimensionless pressure and dimensionless temperature fields, respectively. The dimensionless variables and parameters used in (1)-(3) are given by

$$
\begin{gathered}
a=\frac{2 D}{L}, \\
\bar{r}=\frac{2 r}{D}, \\
\bar{x}=\frac{x}{L}, \\
\bar{u}(\bar{x}, \bar{r})=\frac{u}{u_{o}}, \\
\bar{v}(\bar{x}, \bar{r})=\frac{v}{a u_{o}}, \\
\operatorname{Re}_{r}=\frac{\rho u_{o} D}{\mu}, \\
\operatorname{Pe}_{r}=\frac{\rho c_{p} u_{o} D}{k}=\operatorname{Re}_{r} \operatorname{Pr}, \\
u_{o}=\frac{p_{1}-p_{2}}{32 \mu L / D^{2}}, \\
\bar{p}(\bar{x})=\frac{p-p_{1}}{p_{2}-p_{1}}, \\
\bar{x}, \bar{r})=\frac{T-T_{1}}{q_{s}^{\prime \prime} D /(2 k)},
\end{gathered}
$$

where $u_{o}, p_{1}, p_{2}$, and $T_{1}$ are reference axial velocity, inlet pressure, outlet pressure, and inlet temperature, respectively. $q_{s}^{\prime \prime}$ is the constant heat flux applied at the inner surface of the tube. The boundary conditions of (1)-(3) are given by

$$
\begin{array}{cl}
\bar{x}=0: & \bar{u}_{\mathrm{avg}}=1, \\
\bar{x}=0: & \theta=0, \\
\bar{x}=1: & \bar{p}=1, \\
\bar{r}=0: & \frac{\partial \bar{u}}{\partial \bar{r}}=0, \\
\bar{r}=0: & \frac{\partial \bar{v}}{\partial \bar{r}}=0, \\
\bar{r}=0: & \frac{\partial \theta}{\partial \bar{r}}=0, \\
\bar{r}=1: & \bar{u}=0, \\
\bar{r}=1: & \bar{v}=\bar{v}_{w}(\bar{x}), \\
\bar{r}=1: & \frac{\partial \theta}{\partial \bar{r}}=1,
\end{array}
$$

where $\bar{v}_{w}(\bar{x})=v_{w}(x) /\left(a u_{o}\right), v_{w}(x)$ is the dimensional local suction velocity at the tube inner surface. $u_{\text {avg }}$ is the mean dimensionless axial velocity at any given cross section. Using Kinney [9] and Raithby [11] works, it can be shown that with $0 \leq a \operatorname{Re}_{r} \bar{v}_{w} \leq 1.04$, the convective terms in (2) can be neglected. The aforementioned range results in less than $5.0 \%$ relative error associated with calculating the universal wall friction coefficient by neglecting the convective terms. The previous constraint can practically be satisfied for high aspect ratio tubes $(1 / a \gg 1)$, viscous fluids, or with small suction velocities. Accordingly, the solution of (2) in absence of the convective terms with boundary conditions given by (10a) and (11a) is the following:

$$
\bar{u}(\bar{x}, \bar{r})=2 \bar{p}^{\prime}\left(1-\bar{r}^{2}\right),
$$

where $\bar{p}^{\prime}=d \bar{p} / d \bar{x}$. Substituting (12) in (1) and solving the resulting equation yield to the following distribution of the dimensionless radial velocity:

$$
\bar{v}(\bar{x}, \bar{r})=-\bar{p}^{\prime \prime}\left(\bar{r}-\frac{\bar{r}^{3}}{2}\right),
$$

where $\bar{p}^{\prime \prime}=d^{2} \bar{p} / d \bar{x}^{2}$. Applying the boundary condition given by (11b) results into the following differential equation:

$$
\bar{p}^{\prime \prime}=-2 \bar{v}_{w}(\bar{x}) \text {. }
$$

The mass flow rate $\dot{m}(x)$ and mean axial velocity $u_{\text {avg }}(x)$ can be calculated from the following expressions:

$$
\begin{gathered}
M(\bar{x})=\frac{\dot{m}(x)}{\dot{m}_{o}}=2 \int_{0}^{1} \bar{r} \bar{u} d \bar{r}=\bar{p}^{\prime}(\bar{x}), \\
\bar{u}_{\mathrm{avg}}(\bar{x}) \equiv \frac{u_{\mathrm{avg}}(x)}{u_{o}}=\frac{\dot{m}(x) /\left(\rho \pi D^{2} / 4\right)}{u_{o}}=\bar{p}^{\prime}(\bar{x}),
\end{gathered}
$$

where $\dot{m}_{o}$ is the reference mass flow rate, $\dot{m}_{o}=\rho u_{o} \pi D^{2} / 4$.

The Nusselt number at the tube inner boundary is defined as

$$
\mathrm{Nu} \equiv \frac{h D}{k}=\frac{2}{\theta_{W}-\theta_{m}},
$$

where $h, \theta_{m}$, and $\theta_{W}$ are the convection heat transfer coefficient between the tube inner boundary and the fluid flow $(h=$ $\left.q_{s}^{\prime \prime} /\left[T_{W}-T_{m}\right]\right)$, dimensionless mean bulk temperature, and the dimensionless temperature of the tube inner boundary $\left(\theta_{W}=\theta(\bar{x}, \bar{r}=1)\right)$, respectively. $T_{W}$ and $T_{m}$ are the temperature at the tube inner boundary and the mean bulk temperature of the fluid, respectively. $\theta_{m}$ can be computed from the following equation:

$$
\theta_{m}(\bar{x}) \equiv \frac{T_{m}(x)-T_{1}}{q_{s}^{\prime \prime} D /(2 k)}=4 \int_{0}^{1} \bar{r}\left(1-\bar{r}^{2}\right) \theta(\bar{x}, \bar{r}) d \bar{r} .
$$

The Integral Energy Equation. The integral form of the energy equation can be formed using (3). It can be expressed in the following form:

$$
\bar{u}_{\mathrm{avg}} \frac{d \theta_{m}}{d \bar{x}}+4\left(\frac{\bar{v}_{w}}{\mathrm{Nu}}\right)=\frac{4}{a \mathrm{Pe}_{r}} .
$$


The Fully Developed Nusselt Number. Under fully developed condition, $\partial \theta / \partial \bar{x}=d \theta_{m} / d \bar{x}$. As such, (3) can be reduced to the following using (19):

$$
\begin{aligned}
& 8\left(1-\bar{r}^{2}\right)\left\{1-\frac{a \mathrm{Pe}_{r} \bar{v}_{w}}{\mathrm{Nu}_{\mathrm{fd}}}\right\}+2 a \mathrm{Pe}_{r} \bar{v}_{w}\left(\bar{r}-\frac{\bar{r}^{3}}{2}\right) \frac{\partial \theta_{\mathrm{fd}}}{\partial \bar{r}} \\
& =\frac{2}{\bar{r}} \frac{\partial}{\partial \bar{r}}\left(\bar{r} \frac{\partial \theta_{\mathrm{fd}}}{\partial \bar{r}}\right),
\end{aligned}
$$

where $\mathrm{Nu}_{\mathrm{fd}}$ is the fully developed Nusselt number. Equation (20) can be solved analytically and $\mathrm{Nu}_{\mathrm{fd}}$ expression can be arranged in the following form:

$$
\begin{aligned}
\frac{\mathrm{Nu}_{\mathrm{fd}}}{Z} & \\
= & \left(8\left\{1-\exp \left(\frac{3 Z}{8}\right)\right\}\right. \\
& \left.+2 Z \exp \left(\frac{3 Z}{8}\right) \int_{-3 / 4}^{0} \frac{\exp (Z y / 2)}{\sqrt{y+1}} d y\right) \\
& \quad\left(Z+8\left\{1-\exp \left(\frac{3 Z}{8}\right)\right\}\right. \\
& \left.+2 Z \exp \left(\frac{3 Z}{8}\right) \int_{-3 / 4}^{0} \frac{\exp (Z y / 2)}{\sqrt{y+1}} d y\right)^{-1},
\end{aligned}
$$

where $Z=a \mathrm{Pe}_{r} \bar{v}_{w}(\bar{x})$. The following approximation can be used:

$$
\frac{1}{\sqrt{y+1}} \cong a_{1} \exp \left(a_{2} y\right)+a_{3} \exp \left(a_{4} y\right), \quad-\frac{3}{4} \leq y \leq 0 .
$$

This approximation has maximum relative error less than $0.3 \%$ when the coefficients are equal to

$$
\begin{aligned}
& a_{1}=9.8075 \times 10^{-3} ; \quad a_{2}=-5.3965 ; \\
& a_{3}=0.9874 ; \quad a_{4}=-0.49834 .
\end{aligned}
$$

By substituting (22) in (21), $\mathrm{Nu}_{\mathrm{fd}}$ can be approximated by the following expression:

$$
\begin{aligned}
& \frac{\mathrm{Nu}_{\mathrm{fd}}}{Z} \\
& =\left(8\left\{1-\exp \left(\frac{3 Z}{8}\right)\right\}-A_{1}\left\{\exp \left(a_{5}\right)-\exp \left(\frac{3 Z}{8}\right)\right\}\right. \\
& \left.\quad-A_{2}\left\{\exp \left(a_{6}\right)-\exp \left(\frac{3 Z}{8}\right)\right\}\right) \\
& \quad\left(8\left\{1-\exp \left(\frac{3 Z}{8}\right)\right\}-A_{1}\left\{\exp \left(a_{5}\right)-\exp \left(\frac{3 Z}{8}\right)\right\}\right. \\
& \left.\quad-A_{2}\left\{\exp \left(a_{6}\right)-\exp \left(\frac{3 Z}{8}\right)\right\}+Z\right)^{-1},
\end{aligned}
$$

where

$$
\begin{array}{ll}
a_{5}=-\left(\frac{3}{4}\right) a_{2}, & a_{6}=-\left(\frac{3}{4}\right) a_{4}, \\
A_{1}=\frac{4 a_{1} Z}{2 a_{2}+Z}, & A_{2}=\frac{4 a_{3} Z}{2 a_{4}+Z} .
\end{array}
$$

2.1.1. Case I: Linear Distribution of the Suction Velocity. For this case, the dimensionless suction velocity denoted by $\bar{v}_{w, A}$ has the following linear distribution:

$$
\bar{v}_{w, A}(\bar{x})=B+2\left(\bar{v}_{o}-B\right) \bar{x},
$$

where $\bar{v}_{o}=v_{o} /\left(a u_{o}\right), v_{o}$ is the average suction velocity over the length of tube inner surface. $B$ is an arbitrary controlling parameter.

2.1.2. Case II: Exponential Distribution of the Suction Velocity. For this case, the dimensionless suction velocity denoted by $\bar{v}_{w, B}$ has the following exponential distribution:

$$
\bar{v}_{w, B}(\bar{x})=\bar{v}_{o} \frac{C \exp (C \bar{x})}{\exp (C)-1},
$$

where $C$ is an arbitrary controlling parameter.

2.1.3. The Pressure Gradient for Both Cases. Substituting (26) and (27) in (14) and solving for the dimensionless pressure gradient, the following expressions can be obtained:

$$
\frac{d \bar{p}}{d \bar{x}}= \begin{cases}1-2 \bar{v}_{o} \bar{x}^{2}+2 B\left(\bar{x}^{2}-\bar{x}\right), & \text { Case I, } \\ 1-2 \bar{v}_{o}\left[\frac{\exp (C \bar{x})-1}{\exp (C)-1}\right], & \text { Case II. }\end{cases}
$$

The ranges of $B$ and $\bar{v}_{o}$ that result in having both $\bar{u}_{\mathrm{avg}, A}$ and $\bar{v}_{w, A}$ larger than zero can be shown to be

$$
0 \leq \bar{v}_{o}<\frac{1}{2}, \quad 0 \leq B \leq 2 \bar{v}_{o} .
$$

The ranges of $C$ and $\bar{v}_{o}$ that result in having both $\bar{u}_{\mathrm{avg}, B}$ and $\bar{v}_{w, B}$ larger than zero can be shown to be

$$
0 \leq \bar{v}_{o}<\frac{1}{2}, \quad-\infty<C<\infty
$$

2.2. Perfect Fluid Slip Case (Ideal Case). The ideal case of the present problem is constructed when the fluid is subjected to perfect slip condition at the solid boundary. For this ideal case, the conservation of mass and the continuity equation reveal the following expressions:

$$
\bar{u}_{s}=\left\{\begin{array}{cc}
1-2 \bar{v}_{o} \bar{x}^{2}+2 B\left(\bar{x}^{2}-\bar{x}\right), & \text { Case I, } \\
1-2 \bar{v}_{o}\left\{\frac{\exp (C \bar{x})-1}{\exp (C)-1}\right\}, & \text { Case II, } \\
\bar{v}=\bar{v}_{w} \bar{r}, &
\end{array}\right.
$$


where $\bar{u}_{s}=u_{\mathrm{s}} / u_{o}, u_{s}$ is the fluid velocity for this ideal case. Under this ideal condition, the energy equation reduces to the following:

$$
a \operatorname{Pe}_{r}\left(\bar{u}_{s} \frac{\partial \theta_{s}}{\partial \bar{x}}+\bar{v}_{w} \bar{r} \frac{\partial \theta_{s}}{\partial \bar{r}}\right)=\frac{2}{\bar{r}} \frac{\partial}{\partial \bar{r}}\left(\bar{r} \frac{\partial \theta_{s}}{\partial \bar{r}}\right),
$$

where $\theta_{s}$ is the dimensionless temperature for the ideal case. For fully developed condition where $\partial \theta_{s} / \partial \bar{x}=d \theta_{m} / d \bar{x},(34)$ can be reduced to the following when (19) is implemented:

$$
4\left\{1-\frac{Z}{\mathrm{Nu}_{s, \mathrm{fd}}}\right\}+Z\left(\bar{r} \frac{\partial \theta_{s, \mathrm{fd}}}{\partial \bar{r}}\right)=\frac{2}{\bar{r}} \frac{\partial}{\partial \bar{r}}\left(\bar{r} \frac{\partial \theta_{s, \mathrm{fd}}}{\partial \bar{r}}\right),
$$

where $\mathrm{Nu}_{s, \mathrm{fd}}$ is the fully developed value of the Nusselt number for the ideal case. By solving (35) and application of the boundary condition given by $(10 \mathrm{c}), \mathrm{Nu}_{s, \mathrm{fd}}$ can be found. It is equal to

$$
\frac{\mathrm{Nu}_{s, \mathrm{fd}}}{Z}=\frac{4\{1-\exp (Z / 4)\}}{4\{1-\exp (Z / 4)\}+Z} .
$$

For this case, the dimensionless mean bulk temperature can be found to be equal to

$$
\theta_{m, s}(\bar{x}) \equiv \frac{T_{s, m}(x)-T_{1}}{q_{s}^{\prime \prime} D /(2 k)}=2 \int_{0}^{1} \bar{r} \theta_{s} d \bar{r} .
$$

2.3. Heat Transfer Enhancement Indicators. Let the heat transfer enhancement indicator $\lambda$ be defined as ratio of the tube inner boundary excess temperature at the exit section for the reference case $\left(v_{w}=0\right)$ to that quantity when $v_{w}>0$. Mathematically, the enhancement indicator $\lambda$ is written as

$$
\lambda=\frac{\left.\theta(\bar{x}=1, \bar{r}=1)\right|_{\bar{v}_{w}=0}}{\theta(\bar{x}=1, \bar{r}=1)} .
$$

When $\bar{v}_{w}=0$, the system becomes an impermeable tube confining an internal flow and subjected to uniform heat flux. For this case, the Nusselt number at the exit can be shown to be correlated to $a \mathrm{Pe}_{r}$ according to the following expression:

$$
\begin{aligned}
\mathrm{Nu}(\bar{x}= & \left.1, \bar{v}_{w}=0\right) \\
= & \left(4.38-1.3 \times 10^{-3} a \mathrm{Pe}_{r}+3.9 \times 10^{-4}\left(a \mathrm{Pe}_{r}\right)^{2}\right. \\
& \left.+6.5 \times 10^{-7}\left(a \mathrm{Pe}_{r}\right)^{3}\right) \\
& \cdot\left(1-4.6 \times 10^{-5} a \mathrm{Pe}_{r}+8.6 \times 10^{-5}\left(a \mathrm{Pe}_{r}\right)^{2}\right. \\
& \left.+4.3 \times 10^{-8}\left(a \mathrm{Pe}_{r}\right)^{3}\right)^{-1}
\end{aligned}
$$

with maximum relative error less than $0.3 \%$ when $1 \leq a \mathrm{Pe}_{r} \leq$ 1000 .

The second performance indicator $\eta_{s}$ is defined as ratio of the tube inner boundary excess temperature at the exit section for the perfect fluid slip case to that quantity under no-slip condition. Mathematically, $\eta_{s}$ is written as

$$
\eta_{s}=\frac{\theta_{s}(\bar{x}=1, \bar{r}=1)}{\theta(\bar{x}=1, \bar{r}=1)} .
$$

2.4. Enhancement Indicators for Fully Developed Flow with Uniform Suction. For uniform suction case, the mean velocity inside the tube can be found using (32) by setting $B=\bar{v}_{o}$. It is equal to

$$
\bar{u}_{s}=\bar{u}_{\mathrm{avg}}=1-2 \bar{v}_{o} \bar{x}, \quad \bar{v}_{o}<\frac{1}{2}
$$

By substituting (41) in (19) and solving the resulting equation, the mean bulk temperature distribution can be obtained. It is given by

$$
\theta_{m}(\bar{x})=\ln \left[1-2 \bar{v}_{o} \bar{x}\right]^{2\left(\left[1 / \mathrm{Nu}_{\mathrm{fd}}\right]-1 /\left[a \mathrm{Pe}_{r} \bar{v}_{o}\right]\right)} .
$$

Accordingly, the dimensionless temperature at the exit is equal to

$$
\begin{aligned}
& \theta(\bar{x}=1, \bar{r}=1) \\
& \quad=\theta_{W}(\bar{x}=1)=\ln \left[1-2 \bar{v}_{o}\right]^{2\left(\left[1 / \mathrm{Nu}_{\mathrm{fd}}\right]-1 /\left[a \mathrm{Pe}_{r} \bar{v}_{o}\right]\right)}+\frac{2}{\mathrm{Nu}_{\mathrm{fd}}} .
\end{aligned}
$$

Therefore, the performance indicators $\lambda$ and $\eta_{s}$ for this case are equal to

$$
\begin{aligned}
\lambda_{\mathrm{fd}} & =\frac{96+11 a \mathrm{Pe}_{r}}{\ln \left[1-2 \bar{v}_{o}\right]^{48\left(\left[a \mathrm{Pe}_{r} / \mathrm{Nu}_{\mathrm{fd}}\right]-1 / \bar{v}_{o}\right)}+48\left(a \mathrm{Pe}_{r} / \mathrm{Nu}_{\mathrm{fd}}\right)}, \\
\eta_{s, \mathrm{fd}} & =\frac{\ln \left[1-2 \bar{v}_{o}\right]^{2\left(\left[a \mathrm{Pe}_{r} / \mathrm{Nu}_{s, \mathrm{fd}}\right]-1 / \bar{v}_{o}\right)}+2\left(a \mathrm{Pe}_{r} / \mathrm{Nu}_{s, \mathrm{fd}}\right)}{\ln \left[1-2 \bar{v}_{o}\right]^{2\left(\left[a \mathrm{Pe}_{r} / \mathrm{Nu}_{\mathrm{fd}}\right]-1 / \bar{v}_{o}\right)}+2\left(a \mathrm{Pe}_{r} / \mathrm{Nu}_{\mathrm{fd}}\right)} .
\end{aligned}
$$

The plots of $\theta_{m}(\bar{x}=1), \theta_{s, m}(\bar{x}=1), \mathrm{Nu}_{\mathrm{fd}}, \mathrm{Nu}_{s, \mathrm{fd}}$, and $\lambda_{\mathrm{fd}}$ for various $a \mathrm{Pe}_{r}$ and $\bar{v}_{o}$ values are seen in Figures $2-4$. The upper value of $\bar{v}_{o}$ that makes $\lambda_{\mathrm{fd}}=1$ can be obtained using numerical solving techniques [20]. It is denoted by $\bar{v}_{o, p} \cdot \bar{v}_{o, p}$ can be correlated to $a \mathrm{Pe}_{r}$ through the following correlation:

$$
\begin{aligned}
\bar{v}_{o, p}=( & -2.8076 \times 10^{6}+820470\left(a \mathrm{Pe}_{r}\right) \\
& \left.-83102\left(a \mathrm{Pe}_{r}\right)^{2}+18754\left(a \mathrm{Pe}_{r}\right)^{3}\right) \\
\cdot & \left(1+713480\left(a \mathrm{Pe}_{r}\right)-123760\left(a \mathrm{Pe}_{r}\right)^{2}\right. \\
& \left.+37172\left(a \mathrm{Pe}_{r}\right)^{3}\right)^{-1} .
\end{aligned}
$$

The percentage error associated with (46) is less than $0.62 \%$ when $a \mathrm{Pe}_{r} \geq 4$. The plot of $a \mathrm{Pe}_{r}$ versus $\bar{v}_{o, p}$ is shown in Figure 3. Surprisingly, the value of $\bar{v}_{o}$ making $\eta_{s, \mathrm{fd}}=1$ is found to be independent on $a \mathrm{Pe}_{r}$. This value is denoted by $\bar{v}_{o, e}$ and it is equal to

$$
\bar{v}_{o, e}=0.31606 \text {. }
$$

The plots of $\eta_{s, \mathrm{fd}}$ for various $a \mathrm{Pe}_{r}$ and $\bar{v}_{o}$ are seen in Figure 4 . $\eta_{s, \mathrm{fd}} \geq 1$ when $\bar{v}_{o}>\bar{v}_{o, e}$. 




FIGURE 2: Effects of $\bar{v}_{o}$ on outlet mean bulk temperature under fully developed flow condition.

\section{Numerical Methodology and Results}

Equations (3) and (35) are solvable numerically without iterations using the implicit finite difference method discussed by Khaled [21] and Blottner [22]. They were discretized using two-point backward and three-point central differencing quotients for the first derivative with respect to $\bar{x}$-direction and both first and second derivatives with respect to $\bar{r}$-direction, respectively. The finite difference equation of (3) is given by

$$
\begin{aligned}
& {\left[\frac{\bar{r}_{j}-0.5 \Delta \bar{r}}{\Delta \bar{r}^{2}}+\frac{a \mathrm{Pe}_{r} \bar{r}_{j} \bar{v}_{i, j}}{4 \Delta \bar{r}}\right] \theta_{i, j-1}-\left[\frac{2 \bar{r}_{j}}{\Delta \bar{r}^{2}}+\frac{a \mathrm{Pe}_{r} \bar{r}_{j} \bar{u}_{i, j}}{2 \Delta \bar{x}}\right] \theta_{i, j}} \\
& \quad+\left[\frac{\bar{r}_{j}+0.5 \Delta \bar{r}}{\Delta \bar{r}^{2}}-\frac{a \mathrm{Pe}_{r} \bar{r}_{j} \bar{v}_{i, j}}{4 \Delta \bar{r}}\right] \theta_{i, j+1} \\
& =-\left(\frac{a \mathrm{Pe}_{r} \bar{r}_{j} \bar{u}_{i, j}}{2 \Delta \bar{x}}\right) \theta_{i-1, j} .
\end{aligned}
$$

The pair $(i, j)$ represents the location of the discretized point in the numerical grid of the fluid domain. The number $n$ represents the total number of $j$-nodes along each $i$-section. The tube inner surface is located in the numerical grid at $(i, j=n)$ while the center of the tube is located in the numerical grid at $(i, j=1)$. The resulting $n-1(n=401)$ tridiagonal system of algebraic equations obtained by (48) at a given $i$-section was solved using Thomas algorithm [22]. The previous procedure step was repeated for the consecutive $i$ values until $i$ reached the value $m(m=2001)$ at which $\bar{x}=$ 1. Numerical investigations were performed using different mesh sizes to assess and ascertain grid-size independent results. Furthermore, doubling the values of $m$ and $n$ resulted in less than $0.4 \%$ error in the calculated parameters for moderate $a \mathrm{Pe}_{r}$ values.

The results of the present work are shown in Figures 214. Those obtained using the described numerical method

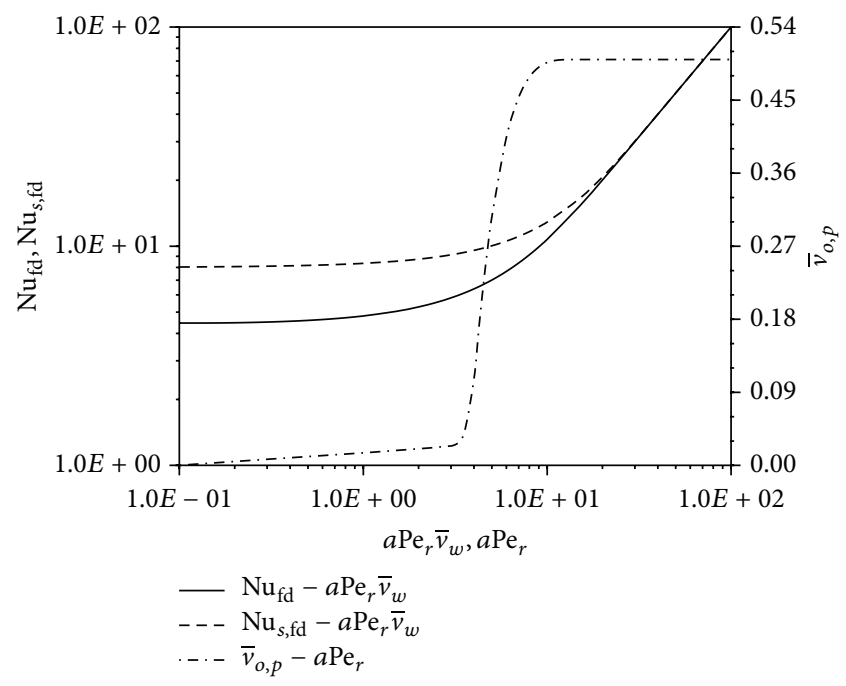

FIGURE 3: Effects of $a \mathrm{Pe}_{r}$ on fully developed Nusselt numbers and $\bar{v}_{o, p}$.

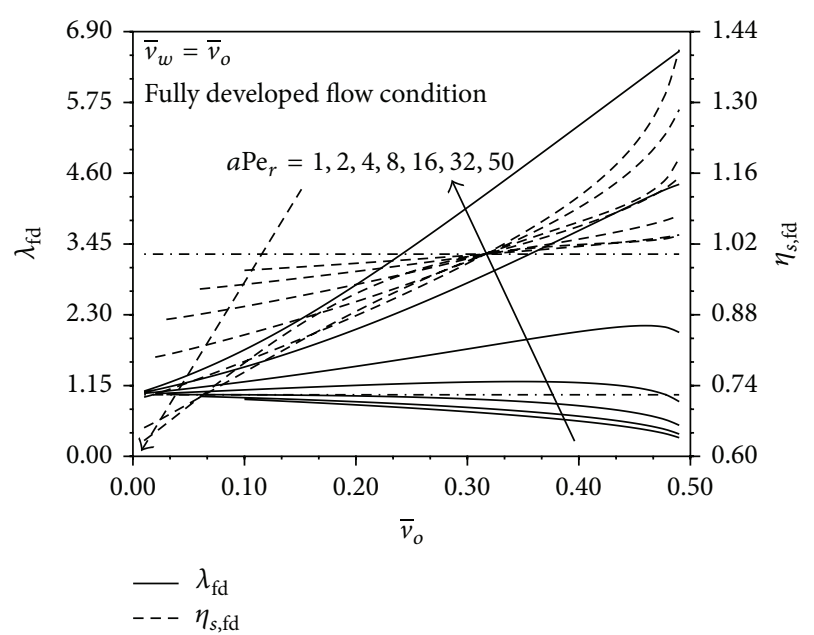

FIGURE 4: Effects of $\bar{v}_{o}$ on the first and second performance indicators under fully developed flow condition.

are shown in Figures 5-14. The numerical results for the case with $a \mathrm{Pe}_{r}=1$ shown in Figures 4-8 were compared with the analytical solution utilizing (17), (24), (26), (27), (38), and (40). The numerical results match well with the analytical solutions since the case with $a \mathrm{Pe}_{r}=1$ results in fully developed flow condition at the tube exit. Also, it is shown from Figure 3 that the fully developed Nusselt number when $a \mathrm{Pe}_{r} \bar{v}_{w}=10$ is equal to $\mathrm{Nu}_{\mathrm{fd}}=10.725$. This quantity can be shown from Figure 9 of Raithby [11] work to be equal to $\mathrm{Nu}_{\mathrm{fd}}=10.83$ when $a \operatorname{Re}_{r} \bar{v}_{w} \rightarrow 0$. The relative error between these values is $0.98 \%$ which is very small. These validations increased the confident levels in the obtained results.

\section{Discussion of the Results}

4.1. The System Thermal Performance for Uniformly Distributed Suction Flow. As suction velocity $v_{w}$ increases, the outlet mean axial velocity $u_{m}(x=L)$ decreases causing 


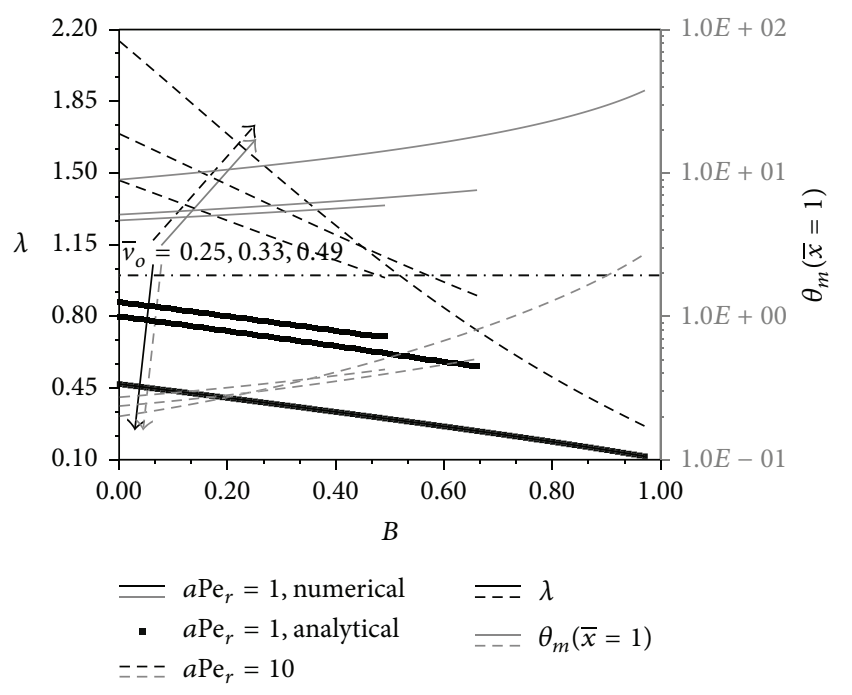

FIGURE 5: Effects of $B$ on performance indicator $\lambda$ and outlet mean bulk temperature for Case I.

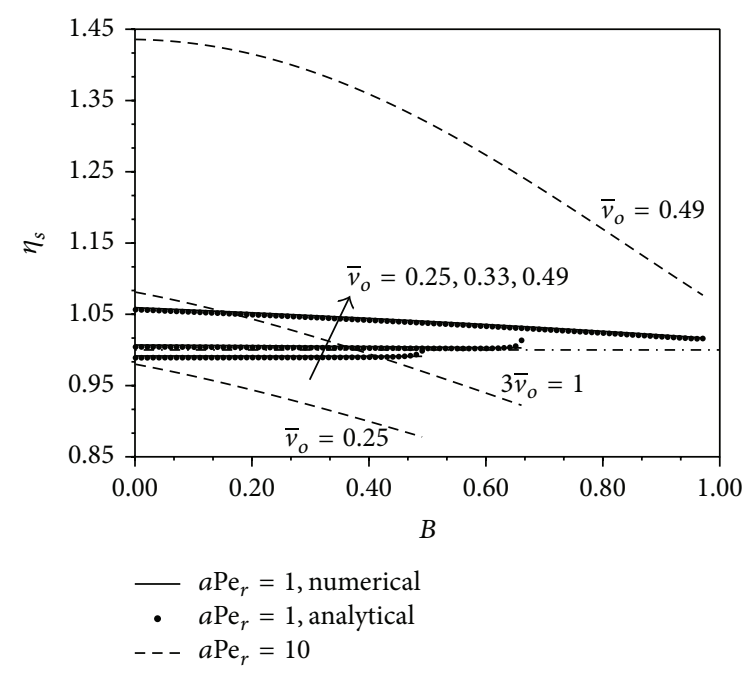

FiguRE 6: Effects of $B$ on performance indicator $\eta_{s}$ for Case I.

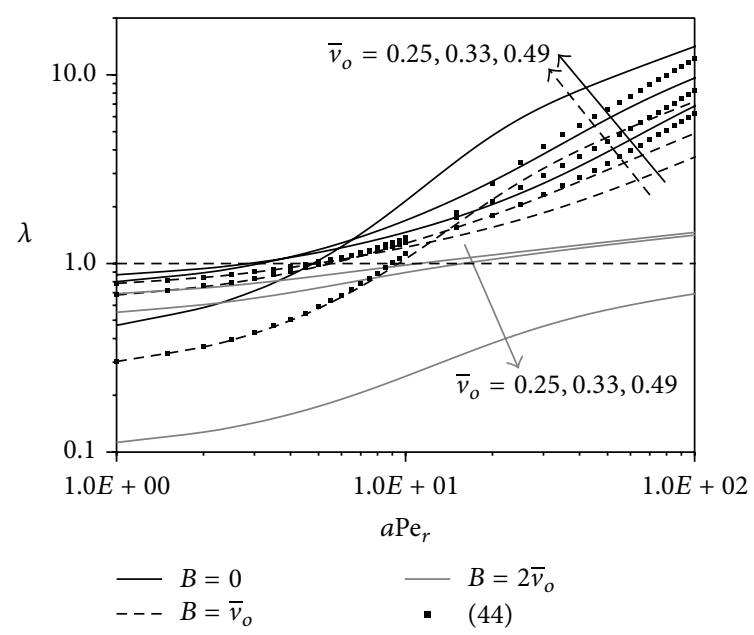

FIGURE 7: Effects of $a \mathrm{Pe}_{r}$ on performance indicators $\lambda$ for various $B$ values for Case I.

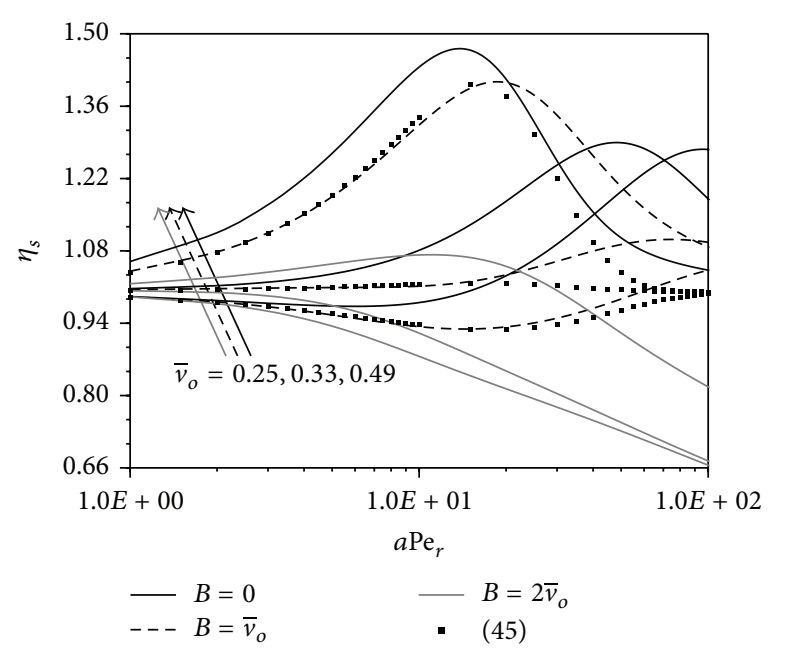

FIGURE 8: Effects of $a \mathrm{Pe}_{r}$ on performance indicators $\eta_{s}$ for various $B$ values for Case I.



FIGURE 9: Effects of $C$ on performance indicator $\lambda$ and outlet mean bulk temperature for Case II.

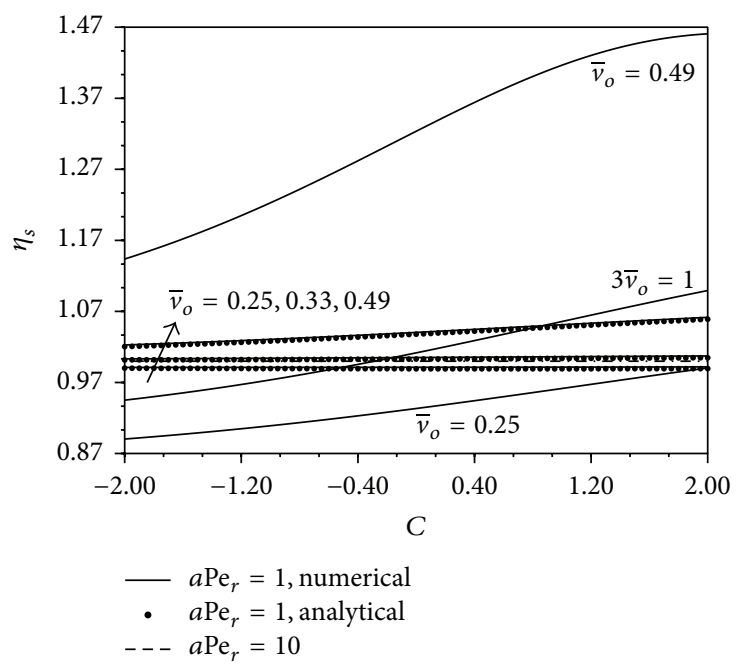

FIgURE 10: Effects of $C$ on performance indicator $\eta_{s}$ for Case II. 


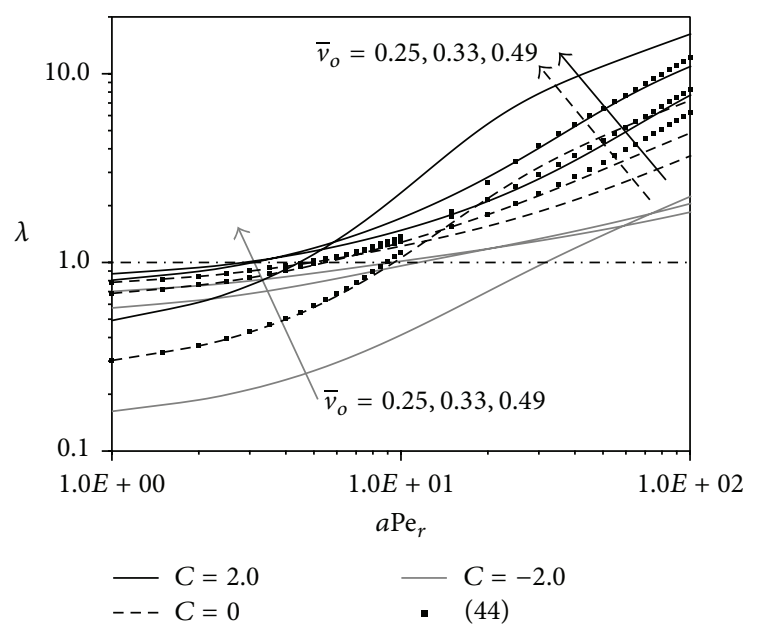

FIGURE 11: Effects of $a \mathrm{Pe}_{r}$ on performance indicators $\lambda$ for various $C$ values for Case II.

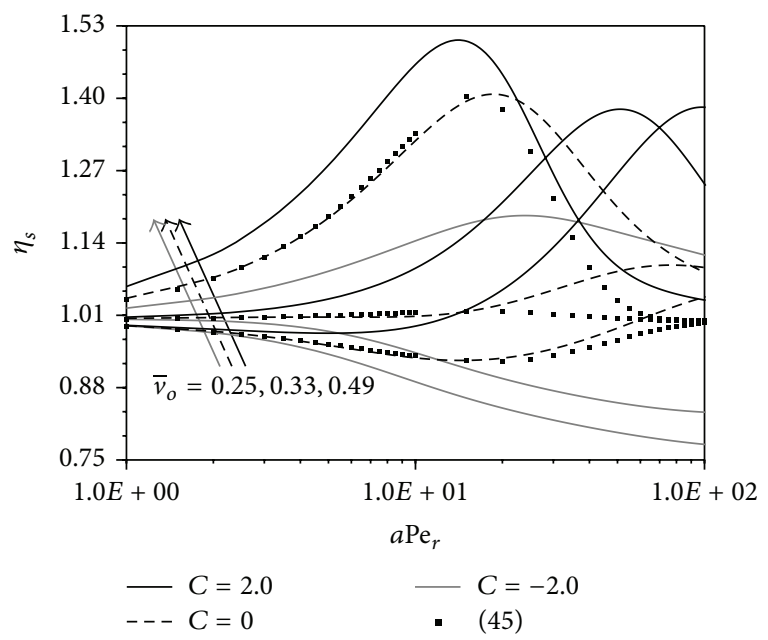

FIGURE 12: Effects of $a \mathrm{Pe}_{r}$ on performance indicators $\eta_{s}$ for various $C$ values for Case II.

the axial convection which is proportional to $a \mathrm{Pe}_{r} \bar{u}_{\mathrm{avg}} d \theta_{m} / \bar{x}$ to decrease. This may cause an increase in the outlet mean bulk temperature $T_{m}(x=L)$ as shown in Figure 2 for small $a \mathrm{Pe}_{r}$ values, $a \mathrm{Pe}_{r} \leq 4$. When $a \mathrm{Pe}_{r} \geq 8$, the transverse convection becomes significant as it is proportional to $2 a \mathrm{Pe}_{r} \bar{v}_{w}\left(\theta_{W}-\theta_{m}\right)$. The latter expression is equivalent to $4 a \mathrm{Pe}_{r} \bar{v}_{w} / \mathrm{Nu}$. This expression increases as $a \mathrm{Pe}_{r} \bar{v}_{o}$ increases. This increase causes significant reduction in the temperature boundary layer thickness which causes reduction in the outlet mean bulk temperature as shown in Figure 2. The sum of the axial and transverse convection is constant which is equal to the total convection heat transfer rate given by $q_{\mathrm{conv}}=$ $q_{s}^{\prime \prime} \pi D L$. When $\bar{v}_{o}$ approaches $\bar{v}_{o}=0.5$, the axial advection which is proportional to $\bar{u}_{\text {avg }}$ approaches zero at the tube outlet. This causes $T_{m}(x=L)$ to increase apparently as $\bar{v}_{o}$ approaches $\bar{v}_{o}=0.5$ asymptotically as can be seen from Figure 2.

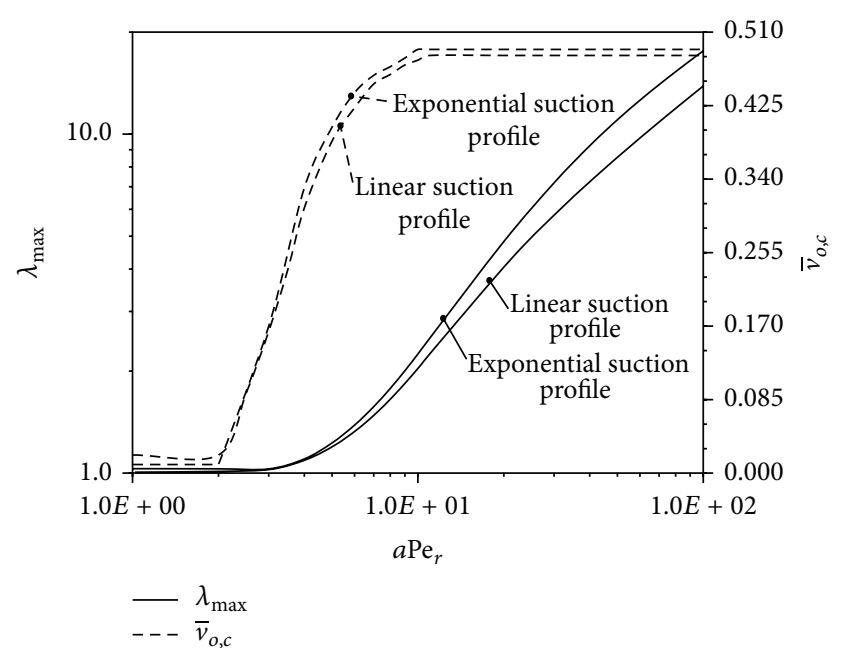

Figure 13: Effects of $a \mathrm{Pe}_{r}$ on $\lambda_{\max }$ and $\bar{v}_{o, c}$ for both Case I and Case II.

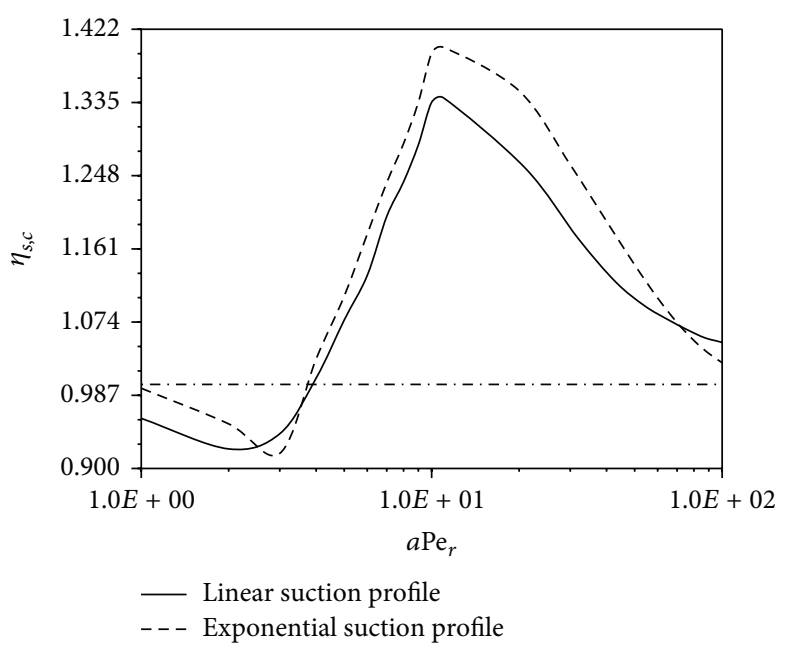

Figure 14: Effects of $a \mathrm{Pe}_{r}$ on $\eta_{s, c}$ for both Case I and Case II.

The Nusselt number for perfect fluid slip at the solid boundary (36) case is larger than that for the no-slip condition case (24) as shown in Figure 3; that is, $\mathrm{Nu}_{s}>\mathrm{Nu}$. This indicates that the tube excess temperature at the boundary for the perfect fluid slip condition is smaller than that for the noslip condition case. That excess temperature is proportional to $\theta_{W}-\theta_{m}$. Consequently, the transverse convection due to fluid slip is smaller than that due to no-slip condition as this convection is proportional to both $a \mathrm{Pe}_{r} \bar{v}_{w}$ and $\theta_{W}-\theta_{m}$. As a result, the axial convection due to fluid slip condition is larger than that due to no-slip condition as the total convection heat transfer rate is constant. Therefore, $T_{m}(x=L)$ for the fluid slip condition is always larger than that for the no-slip condition as shown in Figure 2. It is shown from Figure 3 that the asymptotic value of $\mathrm{Nu}_{\mathrm{fd}}$ is equal to $\mathrm{Nu}_{\mathrm{fd}}=a \mathrm{Pe}_{r} \bar{v}_{w}$. This finding is shown in the works of Kinney [9] and Raithby [11].

Figure 4 shows that the first heat transfer performance indicator $\lambda_{\mathrm{fd}}$ is larger than one when $0<\bar{v}_{o}<\bar{v}_{o, p}$, 
where the variation of $\bar{v}_{o, p}$ with $a \mathrm{Pe}_{r}$ is shown in Figure 3 and given by (46). It can be shown from Figure 3 that when $a \mathrm{Pe}_{r} \geq 9.78, \bar{v}_{o, p} \geq 0.495$. It should be mentioned here that having $\lambda_{\mathrm{fd}}>1$ indicates that the heat transfer to the permeable tube exposed to suction flow results in having lower tube outlet temperature than that for the impermeable tube. Consequently, more thermal energy can be transferred from the permeable tube to the fluid in order to raise its outlet temperature to reach that of the impermeable tube. Furthermore, Figure 4 shows that the second heat transfer performance indicator $\eta_{s, \mathrm{fd}}$ becomes above one when $\bar{v}_{o}>$ $\bar{v}_{o, e}=0.31606$. Having $\eta_{s, \text { fd }}>1$ indicates that the permeable tube subjected to no-slip condition has smaller outlet boundary temperature than that for the permeable tube subjected to perfect fluid slip at the solid boundary. This is not the case for impermeable tubes as $\eta_{s, \mathrm{fd}}<1$ when $\bar{v}_{o}=0$ as shown in Figure 4.

4.2. The System Thermal Performance for Linearly Distributed Suction Flow. When $B=0$, the suction flow increases linearly from $\bar{v}_{w}=0$ at the inlet $(\bar{x}=0)$ to maximum value of $\bar{v}_{w}=2 \bar{v}_{o}$ at the tube outlet $(\bar{x}=1)$. For this case, the thermal entry region effect is maximum as $\bar{u}_{\text {avg }}$ is maximum at inlet. In this region, the axial convection coefficient is substantial as it is the coldest fluid region inside the tube. The thermal entry region influences the flow by causing reductions in the boundary excess temperatures near the inlet. Also, the transverse convection is maximum at the tube outlet when $B=0$ as the suction velocity is maximum there. This decreases the boundary excess temperatures near the tube outlet. As $B$ increases, $\bar{u}_{\text {avg }}$ close to the tube inlet decreases due to the increase in $\bar{v}_{w}$ and $\bar{v}_{w}$ close to the tube outlet decreases. Accordingly, both thermal entry region effect and transverse convection at the tube outlet decrease as $B$ increases. Both effects tend to increase both the boundary excess temperature and outlet mean bulk temperature. As a result, $\theta_{m}(\bar{x}=1)$ increases and $\lambda$ decreases as $B$ increases as shown in Figure 5.

For large $a \mathrm{Pe}_{r}$ values, both thermal entry region effect and the transverse convection at the tube outlet are larger than those for smaller $a \mathrm{Pe}_{r}$ values. Accordingly, $\lambda$ values when $a \mathrm{Pe}_{r}=10$ are larger than those when $a \mathrm{Pe}_{r}=1$ as shown in Figure 5. When $a \mathrm{Pe}_{r}=10$ and $B=0$, it is noticed that $\lambda$ increases as $\bar{v}_{o}$ increases. This is because the outlet mean bulk temperature decreases as $\bar{v}_{o}$ increases for large $a \mathrm{Pe}_{r}$ values as seen from Figure 2. Figure 5 shows that $\lambda$ values for $\bar{v}_{o}=1 / 3$ plot are larger than those for $\bar{v}_{o}=0.49$ plot when $B>0.46$. This indicates that the convection due to thermal entrance region effect as compared to transverse convection increases as $\bar{v}_{o}$ decreases when $B>0.46$ and $a \mathrm{Pe}_{r}=10$. When $a \mathrm{Pe}_{r}=10, \eta_{s}$ is noticed from Figure 6 to decrease as $B$ increases. It is because that both thermal entry region effect and $\bar{v}_{w}$ at the tube outlet decrease as $B$ increases. The former effect is due to the increase in $\bar{v}_{w}$ near the tube inlet as $B$ increases. Both effects tend to increase the excess of $\mathrm{Nu}_{s}$ above $\mathrm{Nu}$. Accordingly, transverse convection due to fluid slip condition decreases due to the decrease in the boundary excess temperature. Consequently, the axial convection due to the fluid slip condition is increased more than that due to the no-slip condition. This causes $\theta_{s, m}$ to increase as compared to $\theta_{m}$. Thus, $\eta_{s}$ decreases as $B$ increases.

Figure 7 shows that $\lambda$ increases as $a \mathrm{Pe}_{r}$ increases. At small $a \mathrm{Pe}_{r}$ values, the transverse convection is negligible, and $\mathrm{Nu}_{s}$ approaches $\mathrm{Nu}$ for large $a \mathrm{Pe}_{r}$ values. These lead to have $\eta_{s}$ approaching one either as $a \mathrm{Pe}_{r}$ decreases towards $a \mathrm{Pe}_{r}=$ 0 or as $a \mathrm{Pe}_{r}$ increases above $a \mathrm{Pe}_{r}=100$. These trends can be seen from Figure 8. For moderate $a \mathrm{Pe}_{r}$ values, the increase in $a \mathrm{Pe}_{r}$ not only causes an increase in the transverse convection but it also results in reducing the boundary excess temperature due to the increases in $\mathrm{Nu}$. This reduction is more distinct for the case of the perfect fluid slip condition as $\mathrm{Nu}_{s}>\mathrm{Nu}$. The combined aforementioned effects can result in reduction of transverse convection as $a \mathrm{Pe}_{r}$ increases for the perfect fluid slip case. It is because the transverse convection is proportional to $a \mathrm{Pe}_{r} \bar{v}_{w}\left(\theta_{W}-\theta_{m}\right)$ as mentioned before. Accordingly, $\eta_{s}$ may have one local maximum at a specific $a \mathrm{Pe}_{r}$ value as clearly shown in Figure 8 for $\bar{v}_{o}=0.49$ plots.

4.3. The System Thermal Performance for Exponentially Distributed Suction Flow. For the upper $C$ value $(C=2)$, the thermal entry region effect is maximum since $\bar{u}_{\text {avg }}$ is maximum at tube inlet because $\bar{v}_{w}$ is minimum there. Also, the transverse convection at the tube outlet is maximum as $\bar{v}_{w}$ is maximum there. These effects tend to decrease the boundary excess temperatures at the tube outlet. As $C$ decreases, $\bar{u}_{\text {avg }}$ close to the tube inlet decreases due to the increase in $\bar{v}_{w}$ and $\bar{v}_{w}$ close to the tube outlet decreases. Accordingly, both thermal entry region effect and transverse convection at the tube outlet decrease as $C$ decreases. Both effects increase both the boundary excess temperature and outlet mean bulk temperature. As a result, the $\theta_{m}(\bar{x}=$ 1) increases and $\lambda$ decreases as $C$ decreases as shown in Figure 9.

For large $a \mathrm{Pe}_{r}$ values, both thermal entry region effect and outlet transverse convection are larger than those for smaller $a \mathrm{Pe}_{r}$ values. Accordingly, $\lambda$ values when $a \mathrm{Pe}_{r}=10$ are larger than those when $a \mathrm{Pe}_{r}=1$ as shown in Figure 9. When $a \mathrm{Pe}_{r}=10$ and $C=2$, it is noticed that $\lambda$ increases as $\bar{v}_{o}$ increases. It is because the outlet mean bulk temperature decreases as $\bar{v}_{o}$ increases for large $a \mathrm{Pe}_{r}$ values as shown from Figure 2. Figure 9 shows that $\lambda$ values for $\bar{v}_{o}=1 / 3$ plot are larger than those for $\bar{v}_{o}=0.49$ plot when $C<0.6$. This indicates that the flow gets more dominated by the thermal entry region effect than by the transverse convection as $\bar{v}_{o}$ decreases when $C<0.6$ and $a \mathrm{Pe}_{r}=10$. Figure 11 shows that $\lambda$ increases as $a \mathrm{Pe}_{r}$ increases. When $a \mathrm{Pe}_{r}=10, \eta_{s}$ is noticed from Figure 10 to decrease as $C$ decreases. It is because both thermal entry region effect and $\bar{v}_{w}$ at the outlet decrease as $C$ decreases. As indicated previously, both effects tend to increase the excess of $\mathrm{Nu}_{s}$ above $\mathrm{Nu}$. Accordingly, the transverse convection due to fluid slip condition decreases due to the decrease in the boundary excess temperature. Consequently, the axial convection due to fluid slip condition is increased more than that due to no-slip condition causing $\theta_{s, m}$ to increase. Thus, $\eta_{s}$ decreases as $C$ decreases. Figure 12 shows that for moderate $a \mathrm{Pe}_{r}$ values $\eta_{s}$ may have one local maximum at a specific $a \mathrm{Pe}_{r}$ value. The physical interpretation for this phenomenon is discussed in Section 4.2. 
4.4. The System Maximum Thermal Performance Indicator. Figure 13 shows that the maximum first performance indicator $\lambda_{\max }$ increases as $a \mathrm{Pe}_{r}$ increases. Also, the critical suction parameter $\bar{v}_{o, c}$ that produces $\lambda_{\max }$ is seen from this figure to increase as $a \mathrm{Pe}_{r}$ increases. Moreover, Figure 13 demonstrates that $\lambda_{\text {max }}$ for the exponential suction flow distribution is always larger than that for the linear suction flow distribution. Two interesting findings can be withdrawn from Figure 14. The first one is that, when $a \mathrm{Pe}_{r}=11$, both suction flow distributions will have their maximum $\eta_{s, C}$ values, where $\eta_{s, C}$ is the value of $\eta_{s}$ at the conditions that produce $\lambda_{\max }$. The second one is that, when $a \mathrm{Pe}_{r}=4$, both suction flow distributions will have $\eta_{s}=\eta_{s, C}=1$. It is shown from Figure 13 that the maximum enhancement ratios are 17.62-fold and 14.67fold above those for impermeable tubes for the exponential and linear suction velocity distributions, respectively. These values are obtained at $a \mathrm{Pe}_{r}=100$. Finally, the phenomenon that there is proper flux distribution that maximizes the heat transfer enhancement indicator agrees with the findings of Khaled [23] and Wang et al. [24]. The flux in the present work is considered to be the mass flux of the suction flow while it is the heat flux in Khaled's [23] work and it is a localized mass flux close to the boundary in Wang et al.s [24] work.

\section{Conclusions}

Flow and heat transfer in permeable tubes subjected to transverse suction flow were analyzed in this work. The continuity, momentum, and energy equations of the internal fluid were solved analytically and numerically. The numerical and the analytical results based on negligible combined entry regions were well matched. Two different suction velocity distributions were considered. They are the linear and exponential distributions. The influence of the average suction velocity, the suction velocity profile, and the Peclet number on the heat transfer enhancement indicators was studied. It was found that heat transfer enhancement over that in impermeable tubes is only attainable if large Peclet numbers are encountered. This enhancement is further increased as suction velocities towards the tube outlet increase and as those towards the tube inlet decrease simultaneously. The enhancement mechanisms were identified and they are expanding the entry regions, increasing the transverse advection, and increasing the downstream excess temperatures under same transverse advection. The maximum reported enhancement ratios in this work are 17.62-fold and 14.67-fold above those for impermeable tubes for the exponential and linear suction velocity distributions, respectively. The average suction velocity that maximizes the heat transfer enhancement indicator increases as the Peclet number increases until it reaches asymptotically its uppermost value at large Peclet numbers. Finally, this work reveals that significant heat transfer enhancement is attainable when the suction flow inside the permeable tube is managed properly.

\section{Nomenclature}

a: Aspect ratio $(a=D /[2 L])$

$B$ : Controlling parameter for suction flow with linear profile; (26)
C: $\quad$ Controlling parameter for suction flow with exponential profile; (27)

$c_{p}: \quad$ Fluid specific heat $(\mathrm{J} / \mathrm{kgK})$

D: $\quad$ Tube inner diameter $(\mathrm{m})$

$h$ : $\quad$ Convection heat transfer coefficient $\left(\mathrm{W} / \mathrm{m}^{2} \mathrm{~K}\right)$

$k$ : $\quad$ Fluid thermal conductivity $(\mathrm{W} / \mathrm{m} \cdot \mathrm{K})$

L: $\quad$ Tube length (m)

$\dot{m}$ : Mass flow rate at given section; (15)

$\dot{m}_{o}$ : Mass flow rate at inlet section; $(\mathrm{kg} / \mathrm{s})$

Nu: $\quad$ Nusselt number $(\mathrm{Nu}=h D / k)$

$\mathrm{Pe}_{r}$ : Reference Peclet number $\left(\mathrm{Pe}_{r}=\rho c_{p} u_{o} D / k\right)$

Pr: $\quad$ Fluid Prandtl number

$p_{1}, p_{2}$ : (Inlet, outlet) fluid pressures $\left(\mathrm{N} / \mathrm{m}^{2}\right)$

$\bar{p}: \quad$ Dimensionless fluid pressure $\left(\mathrm{N} / \mathrm{m}^{2}\right)$

$q_{s}^{\prime \prime}$ : Constant heat flux applied at the tube inner boundary $\left(\mathrm{W} / \mathrm{m}^{2}\right)$

$\mathrm{Re}_{r}$ : Reference Reynolds number

$$
\left(\operatorname{Re}_{r}=\rho u_{o} D / \mu\right)
$$

$r, \bar{r}: \quad$ (Dimensional, dimensionless) radial distance $(\bar{r}=2 r / D)$

$T: \quad$ Fluid temperature field (K)

$T_{1}$ : $\quad$ Inlet fluid temperature (K)

$T_{m}$ : $\quad$ Fluid mean bulk temperature field (K)

$T_{W}: \quad$ Tube inner boundary temperature $(\mathrm{K})$

$u, \bar{u}: \quad$ (Dimensional, dimensionless) axial velocity field $\left(\bar{u}=u / u_{o}\right)$

$u_{s}$ : Axial velocity field under perfect slip condition $(\mathrm{m} / \mathrm{s})$

$\bar{u}_{s}: \quad$ Dimensionless axial velocity field under perfect slip condition $\left(\bar{u}_{s}=u_{s} / u_{o}\right)$

$u_{o}$ : Reference axial velocity; (6c)

$v, \bar{v}: \quad$ (Dimensional, dimensionless) transverse velocity $\left(\bar{v}=v / a u_{o}\right)$

$v_{w}, \bar{v}_{w}:$ (Dimensional, dimensionless) local suction velocity $(\mathrm{m} / \mathrm{s})$

$v_{o}, \bar{v}_{o}$ : (Dimensional, dimensionless) average suction velocity $(\mathrm{m} / \mathrm{s})$

$x, \bar{x}: \quad$ Dimensional and dimensionless axial distances $(\bar{x}=x / L)$.

\section{Greek Symbols}

$\eta_{s}$ : Second heat transfer enhancement indicator; (40)

$\lambda$ : First heat transfer enhancement indicator; (38)

$\mu$ : $\quad$ Fluid dynamic viscosity $\left(\mathrm{Ns} / \mathrm{m}^{2}\right)$

$\theta:$ Dimensionless temperature field; (7b)

$\theta_{s}$ : Dimensionless temperature field under perfect slip condition; (34)

$\theta_{m}$ : Dimensionless mean bulk temperature; (18)

$\theta_{W}$ : Tube dimensionless inner boundary temperature; (17)

$\rho: \quad$ Fluid density $\left(\mathrm{kg} / \mathrm{m}^{3}\right)$. 


\section{Subscripts}

avg: Average value of the quantity

fd: Fully developed value of the quantity

$s$ : Quantity under perfect slip flow at the solid boundary.

\section{Conflict of Interests}

The author declares that there is no conflict of interests regarding the publication of this paper.

\section{Acknowledgments}

This paper was funded by the Deanship of Scientific research (DSR), King Abdulaziz University, Jeddah. The author, therefore, acknowledges with thanks DSR technical and financial support.

\section{References}

[1] O. D. Makinde, S. Khamis, M. S. Tshehla, and O. Franks, "Analysis of heat transfer in Berman flow of nanofluids with Navier slip, viscous dissipation, and convective cooling," Advances in Mathematical Physics, vol. 2014, Article ID 809367, 13 pages, 2014.

[2] A. E. Bergles, "Techniques to enhance heat transfer," in Handbook of Heat Transfer, W. M. Rohsenow, J. P. Hartnett, and Y. I. Cho, Eds., chapter 11, McGraw-Hill, New York, NY, USA, 3rd edition, 1998

[3] D. Westphalen, K. W. Roth, and J. Brodrick, "Heat transfer enhancement," ASHRAE Journal, vol. 48, no. 4, pp. 68-71, 2006.

[4] I. Antonir and A. Tamir, "The effect of surface suction on condensation in presence of a non-condensable gas," Journal of Heat Transfer-Transactions of the ASME, vol. 99, no. 3, pp. 496-499, 1977.

[5] J. Lienhard and V. Dhir, "A simple analysis of laminar film condensation with suction," Transactions ASME-Journal of Heat Transfer, vol. 94, no. 3, pp. 334-336, 1972.

[6] A.-R. A. Khaled, A. M. Radhwan, and S. A. Al-Muaike, "Analysis of laminar falling film condensation over a vertical plate with an accelerating vapor flow," Journal of Fluids Engineering, vol. 131, no. 7, Article ID 0713041, 2009.

[7] M. Ali and F. Al-Yousef, "Laminar mixed convection boundary layers induced by a linearly stretching permeable surface," International Journal of Heat and Mass Transfer, vol. 45, no. 21, pp. 4241-4250, 2002.

[8] S.-C. Wang, C.-K. Chen, and Y.-T. Yang, "Natural convection of non-Newtonian fluids through permeable axisymmetric and two-dimensional bodies in a porous medium," International Journal of Heat and Mass Transfer, vol. 45, no. 2, pp. 393-408, 2001.

[9] R. B. Kinney, "Fully developed frictional and heat-transfer characteristics of laminar flow in porous tubes," International Journal of Heat and Mass Transfer, vol. 11, no. 9, pp. 1393-1401, 1968.

[10] R. J. Pederson and R. B. Kinney, "Entrance-region heat transfer for laminar flow in porous tubes," International Journal of Heat and Mass Transfer, vol. 14, no. 1, pp. 159-161, 1971.

[11] G. Raithby, "Laminar heat transfer in the thermal entrance region of circular tubes and two-dimensional rectangular ducts with wall suction and injection," International Journal of Heat and Mass Transfer, vol. 14, no. 2, pp. 223-243, 1971.
[12] M. M. Sorour and M. A. Hassab, "Effect of sucking the hot fluid film on the performance of flat plate solar energy collectors," Applied Energy, vol. 14, no. 3, pp. 161-173, 1983.

[13] M. M. Sorour, M. A. Hassab, and S. Estafanous, "Developing laminar flow in a semiporous two-dimensional channel with nonuniform transpiration," International Journal of Heat and Fluid Flow, vol. 8, no. 1, pp. 44-54, 1987.

[14] V. I. Bubnovich, N. O. Moraga, and C. E. Rosas, "Numerical forced convection in a circular pipe with nonuniform blowing or suction through the porous wall," Numerical Heat Transfer, Part A: Applications, vol. 33, no. 8, pp. 875-890, 1998.

[15] G. J. Hwang, Y. C. Cheng, and M. L. Ng, "Developing laminar flow and heat transfer in a square duct with one-walled injection and suction," International Journal of Heat and Mass Transfer, vol. 36, no. 9, pp. 2429-2440, 1993.

[16] A.-R. A. Khaled, M. Siddique, N. I. Abdulhafiz, and A. Y. Boukhary, "Recent advances in heat transfer enhancements: a review report," International Journal of Chemical Engineering, vol. 2010, Article ID 106461, 28 pages, 2010.

[17] A. Bejan, Convective Heat Transfer, Wiley, New York, NY, USA, 2nd edition, 1995.

[18] P. H. Oosthuizen and D. Naylor, Introduction to Convective Heat Transfer Analysis, McGraw-Hill, New York, NY, USA, 1999.

[19] A.-R. A. Khaled, "Modeling and computation of heat transfer through permeable hollow-pin systems," Advances in Mechanical Engineering, vol. 2012, Article ID 587165, 12 pages, 2012.

[20] S. C. Chapra and P. R. Canale, Numerical Methods for Engineers, McGraw-Hill, New York, NY, USA, 2009.

[21] A.-R. A. Khaled, "Heat transfer enhancement in a vertical tube confining two immiscible falling co-flows," International Journal of Thermal Sciences, vol. 85, pp. 138-150, 2014.

[22] F. G. Blottner, "Finite difference methods of solution of the boundary-layer equations.", American Institute of Aeronautics and Astronautics, vol. 8, pp. 193-205, 1970.

[23] A.-R. A. Khaled, "Heat transfer enhancement due to properly managing the distribution of the heat flux: exact solutions," Energy Conversion and Management, vol. 53, no. 1, pp. 247-258, 2012.

[24] J. Wang, C. Wu, and K. Li, "Heat transfer enhancement through control of added perturbation velocity in flow field," Energy Conversion and Management, vol. 70, pp. 194-201, 2013. 


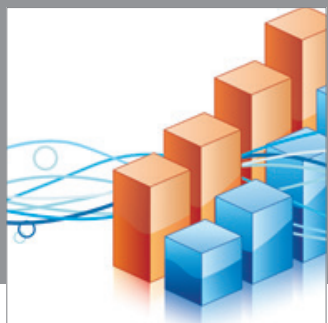

Advances in

Operations Research

mansans

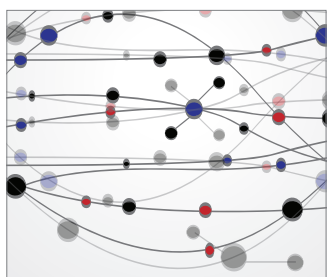

The Scientific World Journal

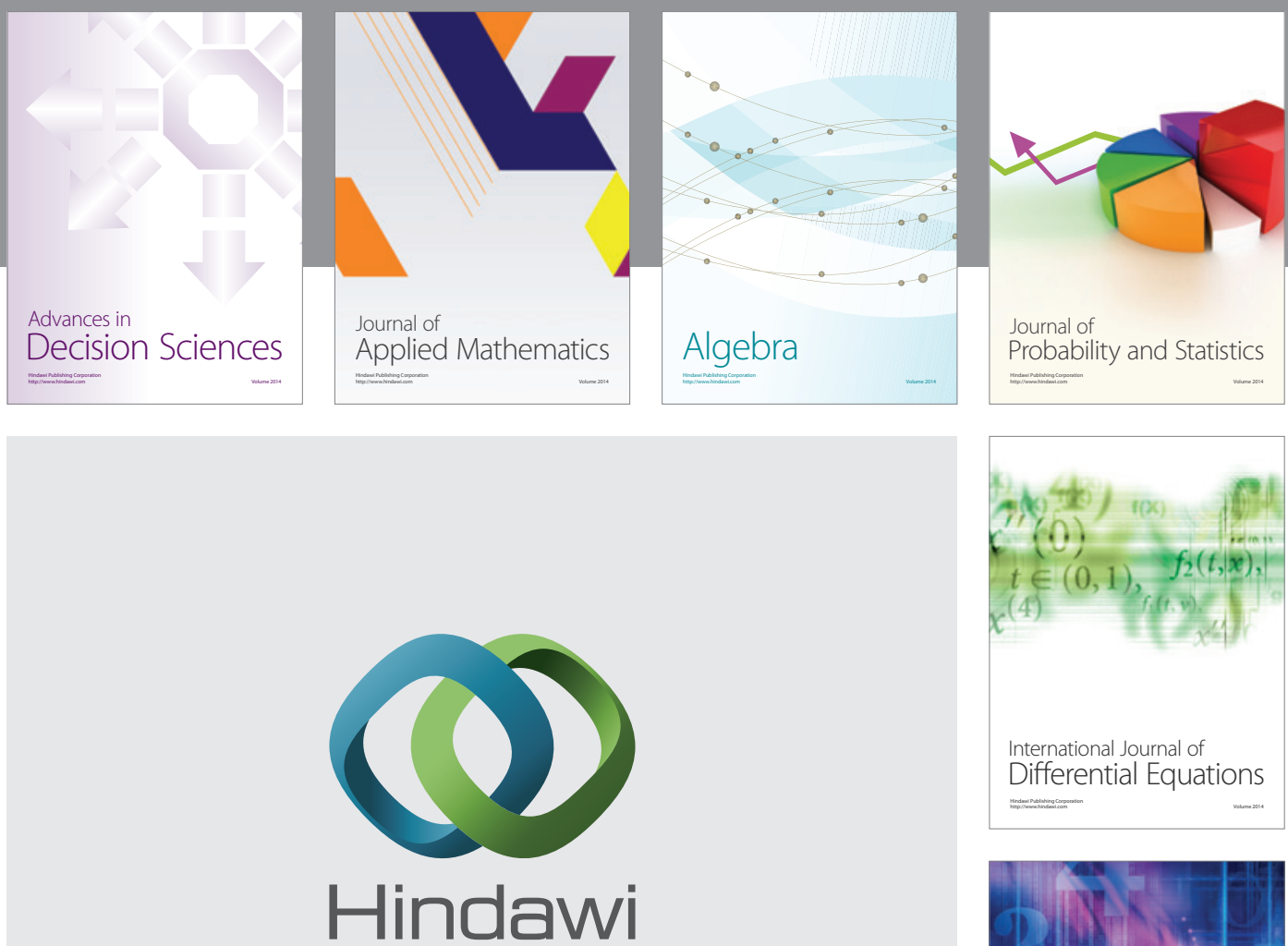

Submit your manuscripts at http://www.hindawi.com
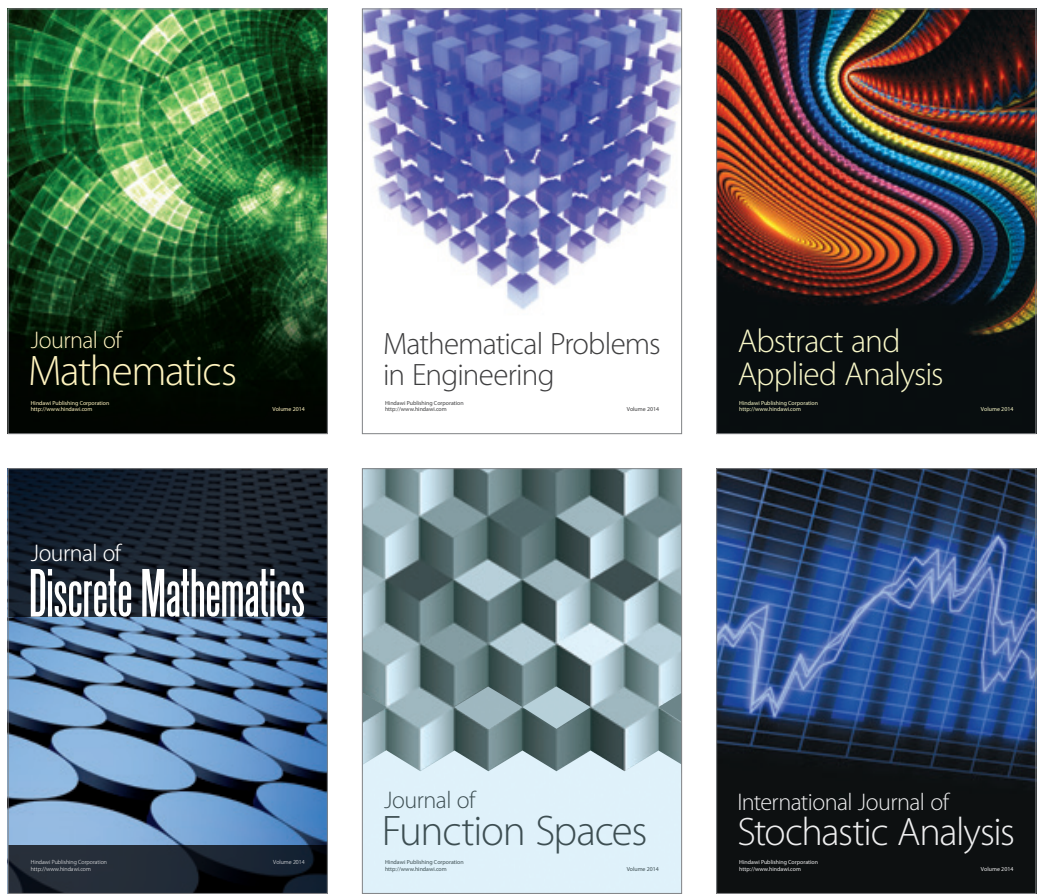

Journal of

Function Spaces




